\title{
Implementation of a P\&O MPPT Algorithm in Low-Milliwatt-Scale Energy Harvesting Wireless Sensor Nodes
}

\author{
Kawsar Ali and Daniel J. Rogers \\ Department of Engineering Science \\ University of Oxford, OX1 3PJ, UK \\ Email: \{kawsar.ali, dan.rogers\} @eng.ox.ac.uk
}

\begin{abstract}
The Fractional Open-Circuit Voltage (FOCV) based Maximum Power Point Tracking (MPPT) method, typically used in low power energy harvesting circuits, is not truly dynamic and wastes harvestable energy in each MPPT cycle. Commercialoff-the-shelf (COTS) energy harvesting chips offer provision for externally setting the MPPT reference using more accurate and dynamic algorithms like Perturb-and-Observe (P\&O), Incremental Conductance (INC) etc., but this can come at the cost of increased power consumption and sensing complexity. To address this issue, a simple low power implementation of the P\&O method in an energy harvesting wireless sensor node (WSN) is presented in this paper. With only two additional analog load switches (SOT23 package), and no extra current or voltage sensor, the proposed method achieves dynamic MPPT with a power overhead of only eight microwatts.
\end{abstract}

\section{INTRODUCTION}

On-board harvesting of ambient energy (light, heat, vibration, radio frequency signals etc.) complemented with a rechargeable energy storage (battery, supercapacitor etc.) has proven to be a competitive alternative to a traditional non-rechargeable-battery for powering wireless sensor nodes (WSN), by increasing their operating lifetime [1], [2]. For a given load power, the combined size of the energy harvester (EH) and rechargeable energy storage is minimised if maximum energy harvesting is ensured from the harvesters.

The maximum power point (MPP) of a source occurs where the product of their terminal voltage and output current is maximum. Energy harvesters like photovoltaic (PV) cells, thermoelectric generators (TEG) etc. are high output impedance sources. The method of extracting maximum power from such sources is called impedance matching or maximum power point tracking (MPPT). This is achieved with an active powerelectronic circuit which dynamically controls the loading on the harvester to track its MPP. Depending on the environment parameters like temperature, irradiance etc., the MPP of a harvester may vary in a wide range. The MPPT method must be able to track this varying point efficiently.

Popular MPPT algorithms like Perturb-and-Observe (P\&O) [3] and Incremental Conductance (INC) [4] use the sensed values of voltage and current of the harvester to compute its MPP. The analogue power management integrated circuits (PMICs) normally used in low power energy harvesting applications are not capable of doing such rather complex computation.
Accurate sensing of harvester output current, which is often of the order of only microamperes, is another complexity to be addressed when implementing $\mathrm{P} \& \mathrm{O}$ and INC algorithms.

A simpler MPPT method, commonly found in many low power Commercial-off-the-shelf (COTS) energy harvesting PMICs, is the use of a constant voltage reference derived from the I-V characteristics of the harvester in its nominal operating condition. For example, in the LTC3105 [5], The MPP voltage is determined by

$$
V_{\mathrm{MPP}}=R_{\mathrm{MPP}} \times 10^{-5}
$$

where $R_{\mathrm{MPP}}$ is an external resistor. It is obvious that with the change of the harvester cell parameters due to external influence, this method may result in an operating point which is far away from the MPP. A better variant of this constantvoltage-reference approach, called the fractional open-circuit voltage (FOCV) method, is taken in many other PMICs [6][8], where the MPP voltage is determined by

$$
V_{\mathrm{MPP}}=k V_{\mathrm{OC}} \text {. }
$$

Here, $k$ is a fraction set by a resistive divider and $V_{\mathrm{OC}}$ is the open-circuit voltage of the harvester. The value of $k$ is usually set to 0.8 for PV cells and 0.5 for TEGs with the assumption that their MPP voltages are typically at about $80 \%$ and $50 \%$ of their open circuit voltages, respectively [9], [10]. In this method, the power drawn from the harvester is momentarily interrupted and the open-circuit voltage of the harvester is measured. The circuit then resumes operation with the harvester terminal voltage regulated at $k V_{\mathrm{OC}}$. This is repeated in every MPPT cycle. While this technique is simple to implement and therefore suited for extreme low power harvesters (microwatts) where computational resources are limited, it wastes some harvestable energy during periodic $V_{\mathrm{OC}}$ sampling. This waste should, in theory, be avoidable as the harvester power increases (milliwatts and above) and more computational resource is available.

The FOCV method is also limited to the assumption of a fixed $k$ value which, in reality, may change with varying operating conditions. For example, the choice of $k=0.8$ is based on the standard test conditions (STC) of $1000 \mathrm{~W} / \mathrm{m}^{2}$ (one sun) of irradiance at an ambient temperature of $25^{\circ} \mathrm{C}$. However, in practice, PV cell MPP voltage can vary in the 
range $60 \%$ to $90 \%$ [11], [12] of the open-circuit voltage depending on the irradiance level and temperature, with the temperature effect being more pronounced.

To maximise the energy extracted from the harvester, a dynamic MPPT algorithm is desirable. Many COTS energy harvesting chips provide the option to externally set $V_{\mathrm{MPP}}$ from a microcontroller unit (MCU) using accurate dynamic algorithms like $\mathrm{P} \& \mathrm{O}$ or INC. However, the additional sensing requirement and the increased power consumption for maintaining a continuous MPP reference make this option less attractive.

This paper proposes a solution to this problem with an innovative and simple low power implementation of the $\mathrm{P} \& \mathrm{O}$ algorithm for an energy harvesting WSN. The on-board MCU, which is an integral part of a WSN (to drive the radio and read the sensor data), is used for the MPP computations. However, unlike the standard $\mathrm{P} \& \mathrm{O}$ implementation, the proposed method does not require sensing of the harvester voltage and current. Instead, it uses only the internal voltage sensor of the MCU to periodically sense the PMIC output voltage. Using this voltage, the average output power of the harvester is estimated and used in the $\mathrm{P} \& \mathrm{O}$ algorithm. No energy is wasted as the periodic sampling of harvester open-circuit voltage is avoided. The power overhead for implementing the proposed method is only a few microwatts.

\section{SYSTEM DESCRIPTION}

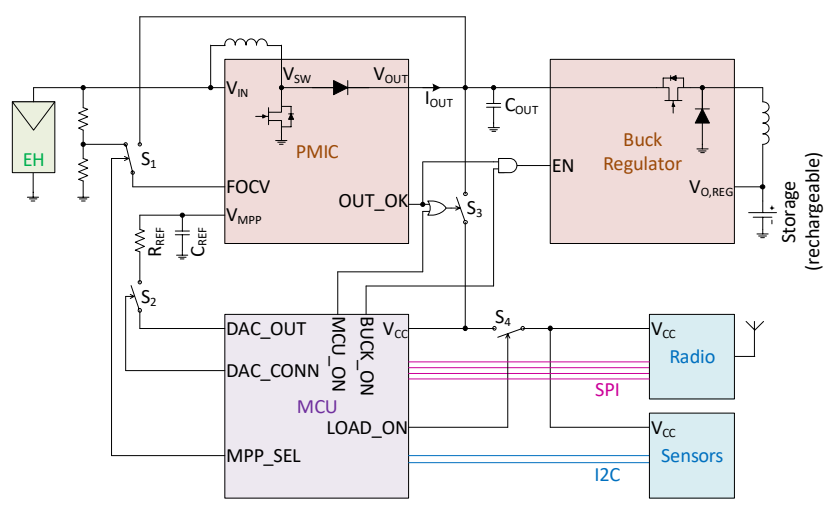

Fig. 1. Functional block diagram of the WSN with the proposed implementation of MPPT.

A simplified functional block diagram of the system is shown in Fig. 1. It is a standalone energy harvesting wireless sensor node comprising an energy harvester $(\mathrm{EH})$, a power management integrated circuit (PMIC), a buck regulator, a rechargeable energy storage (supercapacitor), a microcontroller (MCU), a transceiver (radio), and an array of sensors. The radio and the sensors together are considered as the system load. In many cases, the buck regulator and the radio are integrated within the PMIC [7] and the MCU [13] respectively.

The PMIC boosts the input voltage $V_{\mathrm{IN}}$ into an unregulated output voltage $V_{\text {OUT }}$ with a resistor-programmable upper bound $V_{\text {OUT,OV. A }}$. bulk storage capacitor $C_{\text {OUT }}$ is connected to $V_{\text {OUT }}$ to accumulate enough energy to support the primary load of the system i.e. periodic sensing and radio transmission. Any excess energy from $C_{\text {OUT }}$ is then passed on to the buck regulator which generates a regulated output voltage $V_{\mathrm{O}, \mathrm{REG}}$ which charges the supercapacitor. The body diode of the buck MOSFET helps to support the system load from the charged supercapacitor in the absence of input power from the harvester.

The OUT_OK signal from the PMIC indicates the state of $V_{\text {OUT }}$. It goes high when $V_{\text {OUT }}$ surpasses $V_{\text {OKH }}$ during the charging of $C_{\mathrm{OUT}}$, and goes low when $V_{\mathrm{OUT}}$ falls below $V_{\mathrm{OKL}}$ during the discharging of $C_{\mathrm{OUT}}$. The values of $V_{\mathrm{OKH}}$ and $V_{\mathrm{OKL}}$ are resistor-programmable. The logic OR and the logic AND gates shown in Fig. 1 helps the PMIC and the MCU in the overall operation and energy management of the system. This has been explained in detail in our previous work [14].

The FOCV MPPT [6]-[8] is implemented by periodically turning off the PMIC and then sampling the open-circuit voltage of the harvester. A fixed fraction (determined by the resistive divider) of this open-circuit voltage is used as the MPP reference voltage $V_{\mathrm{MPP}}$ and held in the capacitor $C_{\mathrm{REF}}$ during the period between two such sampling events. The PMIC, while on, simply tracks $V_{\text {MPP }}$ to regulate its input voltage at the MPP.

To implement the proposed MCU-assisted MPPT method, we have introduced only two extra components - an SPDT switch $S_{1}$ and an SPST switch $S_{2}$. Switch $S_{1}$ is used for changing over from FOCV MPPT to MCU-assisted MPPT, and vice versa. Note that in MCU-assisted MPPT we disable the periodic sampling of the harvester open-circuit voltage (thus prevent wastage of harvestable energy), which is achieved by pulling up the FOCV pin to $V_{\text {OUT }}$ in our system (see Fig. 1). Switch $S_{2}$ prevents the slow discharge of $C_{\mathrm{REF}}$ into the DAC_OUT pin of the MCU, after $C_{\mathrm{REF}}$ has been charged to $V_{\mathrm{MPP}}$. A high value resistance $R_{\mathrm{REF}}(\approx 10 \mathrm{M} \Omega)$ is used in series with $C_{\mathrm{REF}}$ to limit its charging current.

As common with low power WSN applications we make extensive use of the low power modes (LPM) [15], [16] of the MCU, where the central processing unit (CPU) and the individual peripherals can be turned-off selectively to minimize energy consumption when the MCU is sleeping. The roles of the general purpose input/output (GPIO) pins shown in Fig. 1 are summarised below.

- MCU_ON: is OR-ed with OUT_OK to keep the MCU on when $V_{\mathrm{OKL}}>V_{\mathrm{OUT}}>V_{\mathrm{CC}, \mathrm{MIN}}$. Here, $V_{\mathrm{CC}, \mathrm{MIN}}$ is the minimum supply voltage required for the MCU, which is typically $1.8 \mathrm{~V}$. The MCU can be turned off only when both OUT_OK and MCU_ON goes low.

- BUCK_ON: is AND-ed with OUT_OK to ensure that the Buck regulator is disabled when $V_{\mathrm{OKL}}>V_{\mathrm{OUT}}>$ $V_{\mathrm{CC}, \mathrm{MIN}}$, even if BUCK_ON is high. This prevents the sudden turn-off of the MCU during the trickle-charging cycles of the rechargeable storage (supercapacitor) on the buck regulator output bus $\left(V_{\mathrm{O}, \mathrm{REG}}\right)$.

- LOAD_ON: controls the switch $S_{4}$ which is used for turning on/off the load (i.e. the radio and the sensors). 
- MPP_SEL: controls the switch $S_{1}$ which is used for selection of MPPT mode between FOCV (MPP_SEL = $0)$ and MCU-assisted (MPP_SEL $=1$ ).

- DAC_CONN: controls the switch $S_{2}$ which is used for making or breaking the connection between $R_{\mathrm{REF}}$ and the DAC_OUT pin.

\section{PROPOSED IMPLEMENTATION OF MPPT}

A. Workflow in the System

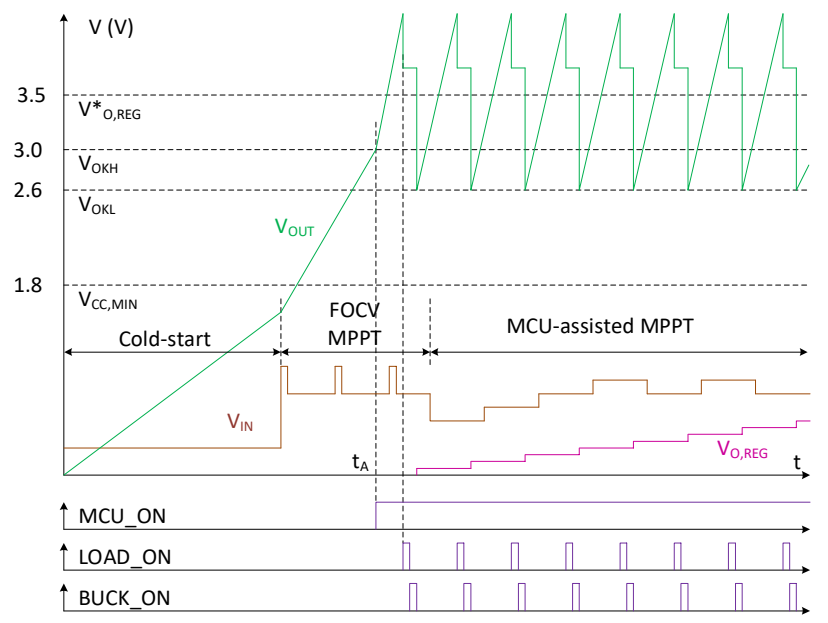

Fig. 2. Illustration of slow buildup of output voltages showing the transition from FOCV MPPT to the proposed MCU-assisted MPPT method. The time axis is not to scale..

Fig. 2 shows an illustration of the voltage build-up in the unregulated and the regulated output buses for our example case discussed later in section V. Note that the numeric values of different voltage levels in Fig. 2 are specific to this study, but the proposed method can be adopted to any other similar application as long as the following relation is satisfied.

$$
V_{\mathrm{O}, \mathrm{REG}}^{*}>V_{\mathrm{OKH}}>V_{\mathrm{OKL}}>V_{\mathrm{CC}, \mathrm{MIN}} .
$$

The major steps of the algorithm are described below.

1) The PMIC starts up in cold-start mode where all of its features are disabled except an auxiliary unregulated hysteretic boost converter whose only function is to charge up $C_{\text {OUT }}$ to a minimum voltage required to power up the main boost converter [6].

2) The cold-start circuit is disabled when the main boost circuit is powered up. The output capacitor $C_{\text {OUT }}$ is now charged by the main boost converter using FOCV MPPT method.

3) When the OUT_OK signal goes high for the first time (with $V_{\mathrm{OUT}}=V_{\mathrm{OKH}}=3 \mathrm{~V}$ at $t_{\mathrm{A}}$ in Fig. 2), the MCU is turned on, and it sets its MCU_ON signal high. Note that $V_{\mathrm{CC}}$ becomes equal to $V_{\mathrm{OUT}}$ after the turn-on of the MCU.

4) The MCU then goes to sleep (i.e. low power mode) for $t_{1}$ s. After waking up, the MCU checks if $V_{\mathrm{CC}}>V_{\mathrm{O}, \mathrm{REG}}^{*}$.

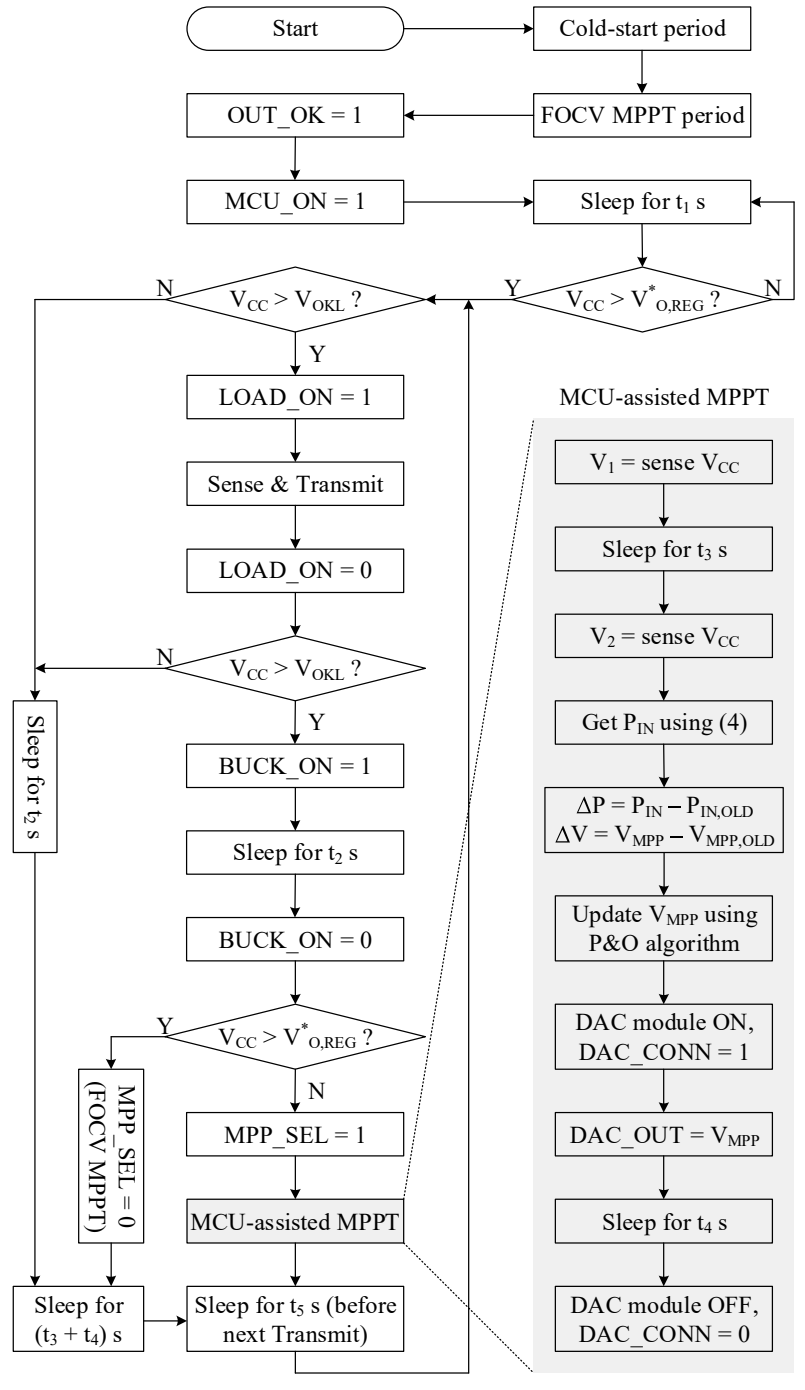

Fig. 3. Flowchart of proposed implementation of MPPT.

If not, it goes back to sleep again for $t_{1} \mathrm{~s}$. This keeps on repeating until $V_{\mathrm{CC}}$ becomes greater than $V_{\mathrm{O}, \mathrm{REG}}^{*}$, when the MCU enters the infinite loop.

5) The infinite loop starts by ensuring $V_{\mathrm{CC}}>V_{\mathrm{OKL}}$. Since sensing and transmitting is the first priority of the system, the LOAD_ON signal is set high so that the radio and the sensors can perform their tasks. The LOAD_ON switch is pulled low when this is done.

6) If $V_{\mathrm{CC}}$ is still more than $V_{\mathrm{OKL}}$ after the radio transmission, the buck regulator is enabled by setting BUCK_ON signal high to charge the rechargeable energy storage (supercapacitor), and the MCU goes to sleep for $t_{2} \mathrm{~s}$. The buck regulator is disabled when either $V_{\text {OUT }}$ falls below $V_{\mathrm{OKL}}$, or the MCU pulls the BUCK_ON signal low after waking up. This is taken care of by the logic AND operation between OUT_OK and BUCK_ON signals. The supercapacitor is thus trickle-charged in each 
iteration.

7) At this point if $V_{\mathrm{CC}} \leq V_{\mathrm{O}, \mathrm{REG}}^{*}$, the MPP_SEL signal is set high to enable the MCU assisted MPPT method, as described in the next subsection. However, if $V_{\mathrm{CC}}>$ $V_{\mathrm{O}, \mathrm{REG}}^{*}$, it is decided that the supercapacitor has been fully charged up to its target voltage $V_{\mathrm{O}, \mathrm{REG}}^{*}$ and the MPP_SEL signal is pulled low to fall back to the FOCV MPPT mode.

8) Finally, the MCU goes into another sleep period for $t_{5}$ $\mathrm{s}$ before going back to the next sense and transmit event (step 5).

Fig. 3 shows the flowchart of the proposed algorithm. It is easy to see that the system prioritises sense and transmit operation, and can continue to support it with energy from only the supercapacitor storage in the absence of harvester energy (e.g. at night for a PV-based WSN) until the supercapacitor voltage falls below $V_{\mathrm{OKL}}$.

\section{B. MCU-assisted MPPT}

After the sense and transmit step (step 5) and the supercapacitor trickle-charge step (step 6) have been completed, the input energy from the harvester only charges the output capacitor $C_{\text {OUT }}$. Assuming the quiescent currents and leakage currents of various parts of the system as constant and negligible, the average input power is estimated as

$$
P_{\mathrm{IN}}=\frac{1}{\eta t_{3}} \int_{V_{1}}^{V_{2}} C_{\text {OUT }}(V) V \cdot \mathrm{d} V
$$

where $V_{1}$ and $V_{2}$ are the sensed values of $V_{\mathrm{CC}}$ (and therefore of $V_{\text {OUT }}$ ) using MCU internal voltage sensor with a fixed time interval $t_{3}$ between the two sensing events, and $\eta$ is the nominal efficiency of the PMIC. Note that the value of $C_{\text {OUT }}$ is a function of its dc bias voltage [17] since it is typically a class II (X7R, X5R etc.) ceramic capacitor. This function is estimated from the datasheet of the capacitor using a linear fit, as shown later in (6) for our example system.

The changes in input power and input reference voltage with respect to their previous iteration values are now calculated as

$$
\begin{gathered}
\Delta P=P_{\mathrm{IN}}-P_{\mathrm{IN}, \mathrm{OLD}} . \\
\Delta V=V_{\mathrm{MPP}}-V_{\mathrm{MPP}, \mathrm{OLD}} .
\end{gathered}
$$

Using $\Delta P$ and $\Delta V$ from above equation a basic $\mathrm{P} \& \mathrm{O}$ algorithm is implemented [3] and the value of $V_{\text {MPP }}$ is updated.

To charge $C_{\mathrm{REF}}$ to the updated $V_{\mathrm{MPP}}$ voltage, the DAC module of the MCU is enabled and the DAC_CONN signal is set high so that the DAC_OUT pin is connected to $R_{\text {REF }}$. The DAC_OUT pin is now held at $V_{\mathrm{MPP}}$ for $t_{4}$ s to complete the charging of $C_{\mathrm{REF}}$. Once completed, the DAC_CONN signal is pulled low and the DAC module is disabled to save power.

Note that the switch $S_{2}$, which is connected between the DAC_OUT pin and $R_{\text {REF }}$ and controlled by the DAC_CONN signal (see Fig. 1), is critical for this application to keep $V_{\mathrm{MPP}}$ steady for the duration between MPPT cycles, which is usually more than $10 \mathrm{~s}$. The MCU pins typically have a leakage current of $50 \mathrm{nA}$ even in their high-impedance state (tri-state). If the
DAC_OUT pin is directly connected to $R_{\mathrm{REF}}$, this leakage current can discharge $C_{\mathrm{REF}}$ (usually a few tens of nanofarads) within fraction of a second. The switch $S_{2}$ realised by a load switch with sub-nanoampere leakage current in its off-state (e.g. TPS22860) holds $V_{\text {MPP }}$ at a steady value for several tens of seconds.

\section{DESIGN ASPECTS}

\section{A. Output Capacitance}

In the energy budget of the system, the sensing and radio transmission has the first priority, then any extra energy is used for charging the supercapacitor. Clearly, $C_{\mathrm{OUT}}$ has to be large enough to sustain at least one sense and transmission cycle [14], and therefore, is calculated as

$$
C_{\mathrm{OUT}}=I_{\mathrm{LOAD}} t_{\mathrm{LOAD}} /\left(V_{\mathrm{OKH}}-V_{\mathrm{OKL}}\right)
$$

where $t_{\mathrm{LOAD}}$ is the combined duration of the sense and transmit events, and $I_{\mathrm{LOAD}}$ is the average current consumed by the load during $t_{\mathrm{LOAD}}$.

For example, in our experiment described in the next section, we need a load current of $20 \mathrm{~mA}$ for a pulse duration of $40 \mathrm{~ms}$. According to (5), for a $V_{\mathrm{OKL}}$ and $V_{\mathrm{OKH}}$ of $2.6 \mathrm{~V}$ and $3 \mathrm{~V}$ respectively, we need at least $2 \mathrm{mF}$ of $C_{\text {OUT }}$ to drive this load. Ceramic capacitors are generally preferred as $C_{\text {OUT }}$ in low power WSN applications as they have very low leakage current. However, among the commercially available ceramic capacitors rated for voltage above $3 \mathrm{~V}$, the highest capacitance value we could find is with the AMK432BJ477MM capacitor, rated as $470 \mu \mathrm{F}, 4 \mathrm{~V}$. Also, the effective value of capacitance of the class II ceramic capacitor (X7R, X5R etc.) drops drastically with the increase of dc bias [17]. We have used 10 numbers of AMK432BJ477MM capacitors in parallel. A simple linear fit of its dc bias characteristics from the datasheet is

$$
C_{\text {OUT }}\left(V_{\mathrm{X}}\right)=\left(-771 V_{\mathrm{X}}+5194\right) \times 10^{-6} .
$$

Equation (6) is used for calculating the effective value of $C_{\text {OUT }}$ in (4).

\section{B. Sleeping Times}

As shown in Fig. 3, the MCU goes to sleep mode (LPM) at various stages of the workflow for various amounts of times. Most of their values $\left(t_{1}, t_{2}, t_{3}, t_{5}\right)$ are application specific and depend on available energy, load power requirement, period of load pulse etc.

Time $t_{4}$ is calculated based on the time constant of the RC network formed by $R_{\mathrm{REF}}$ and $C_{\mathrm{REF}}$. To give enough margin, we chose $t_{4}$ as twice this time constant in our design:

$$
t_{4}=2 \times R_{\mathrm{REF}} C_{\mathrm{REF}} .
$$

The period of the load pulse is given by

$$
T=t_{2}+t_{3}+t_{4}+t_{5}
$$

Note from Fig. 3 that $T$ remains the same irrespective of whether the system is in FOCV MPPT mode or MCU-assisted MPPT mode. For better MPPT accuracy, we could split $t_{3}$ 
into multiple parts and/or eliminate $t_{5}$ completely in order to have multiple MPP update cycles within one load pulse period. However, we must trade any gains against the increased energy consumption that will result.

As an example, the values used in the experimental validation of this work are $t_{1}=1 \mathrm{~s}, t_{2}=1 \mathrm{~s}, t_{3}=10 \mathrm{~s}, t_{4}=200$ $\mathrm{ms}$, and $t_{5}=4.8 \mathrm{~s}$.

\section{Harvester Capacity}

A minimum average power required from the harvester can be calculated for the bare minimum function of the system, which is to sustain the periodic sense and transmit cycle. For a known input voltage $V_{\mathrm{IN}}$, the minimum average input current required from the harvester can be found as

$$
I_{\mathrm{IN}, \mathrm{MIN}}=I_{\mathrm{OUT}, \mathrm{MIN}}\left(V_{\mathrm{OKH}}+V_{\mathrm{OKL}}\right) /\left(2 \eta V_{\mathrm{IN}}\right)
$$

with

$$
I_{\mathrm{OUT}, \mathrm{MIN}}=C_{\mathrm{OUT}}\left(V_{\mathrm{OKH}}-V_{\mathrm{OKL}}\right) / T
$$

For a meaningful implementation of the proposed MPPT method, we must avoid reaching the over-voltage limit $V_{\text {OUT,OV }}$ on the output capacitor during the fixed time interval $t_{3}$ between the two sensing of $V_{\mathrm{CC}}$ events. Therefore, for a known input voltage $V_{\mathrm{IN}}$, the maximum average input current required from the harvester can be found as

$$
I_{\mathrm{IN}, \mathrm{MAX}}=I_{\mathrm{OUT}, \mathrm{MAX}}\left(V_{\mathrm{OUT}, \mathrm{OV}}+V_{\mathrm{OKL}}\right) /\left(2 \eta V_{\mathrm{IN}}\right)
$$

with

$$
I_{\mathrm{OUT}, \mathrm{MAX}}=C_{\mathrm{OUT}}\left(V_{\mathrm{OUT}, \mathrm{OV}}-V_{\mathrm{OKL}}\right) / t_{3}
$$

\section{VALIDATION}

\section{A. Example System}

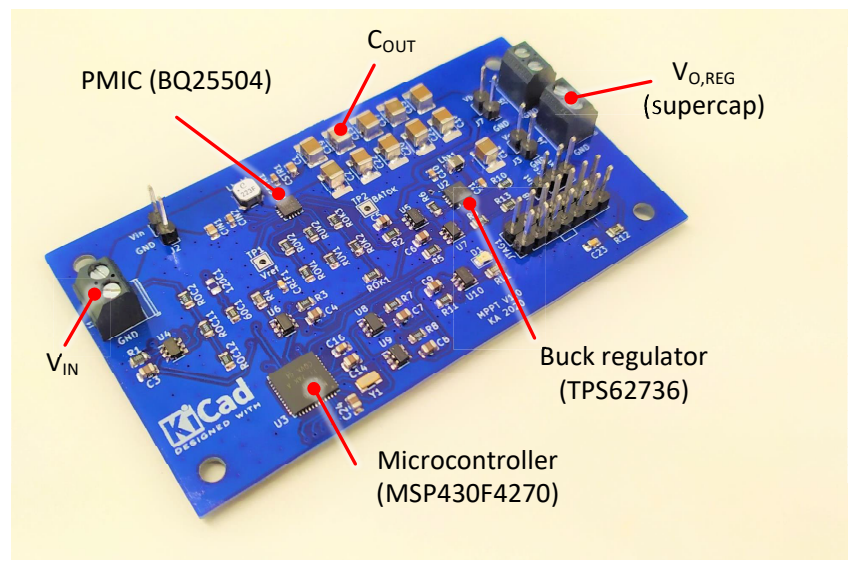

Fig. 4. Experimental setup of the proposed system.

An experimental setup as shown in Fig. 4 was built to validate the proposed concept with a $1 \mathrm{~cm}^{2} \mathrm{PV}$ cell (KXOB2204X3F) used as the energy harvester. The setup uses BQ25504 [6] as the PMIC, TPS62737 as the buck regulator, and MSP430F4270 [15] as the MCU, all from Texas Instruments. The pulsed load of sense and transmit (i.e. sensor and radio respectively in Fig. 1) is emulated by a simple LED in series with a resistor after the load switch $S_{4}$. This is done to keep the system simple and focus on the MPPT part without loss of generality. A $0.33 \mathrm{~F}$ supercapacitor is chosen as the rechargeable storage.

The list of important components used in the experimental setup is presented in Table I. An effort was made to choose the components with the lowest quiescent and leakage currents.

TABLE I

LIST OF IMPORTANT COMPONENTS

\begin{tabular}{ll}
\hline Component & Part number \\
\hline PMIC & BQ25504 \\
Buck regulator & TPS62737 \\
MCU & MSP430F4270 \\
AND gate & $2 \times$ TPS22860 in series \\
OR gate & $2 \times$ TPS22860 in parallel \\
$S_{2}, S_{3}, S_{4}$ (SPST switch) & TPS22860 \\
$S_{1}$ (SPDT switch) & TMUX1119 \\
$C_{\text {OUT }}$ & $10 \times$ AMK432BJ477MM in parallel \\
Rechargeable storage & SCMR14L334SSBB0 $(0.33$ F supercapacitor $)$ \\
\hline
\end{tabular}

\section{B. Experimental Results}

Fig. 5 shows the key waveforms of operation with the convergence of MPPT and the slow charging of the supercapacitor. The FOCV MPPT which is an inbuilt feature of the BQ25504 PMIC is visible briefly in Fig. 5, and with more details in Fig. 6. Two notable load events (sense \& transmit, and buck regulator $\mathrm{ON}$ ) are captured in Fig. 7.

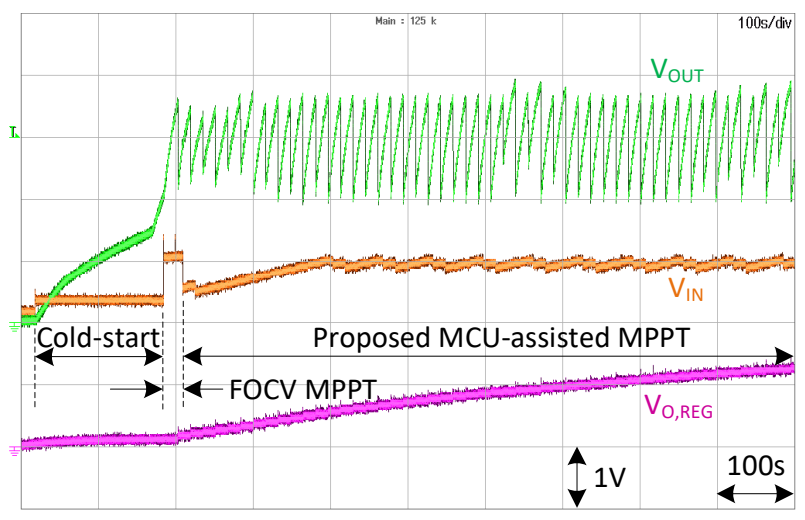

Fig. 5. Key experimental waveforms showing different stages of MPPT and slow charging of the supercapacitor.

A fixed step size of $50 \mathrm{mV}$ is used for the $\mathrm{P} \& \mathrm{O}$ algorithm, which can be smaller or variable for better tracking. Also, the initial guess of the input voltage reference is chosen arbitrarily as $0.5 \mathrm{~V}$. In practice, with prior knowledge of the harvester cell type (e.g. PV cell or TEG etc.) and rating, one can choose this initial guess more precisely to expedite the MPPT convergence.

\section{Power Overhead}

Table II lists the energy required to implement the proposed MPPT method [15]. Since the cycle repeats every $16 \mathrm{~s}$ the 


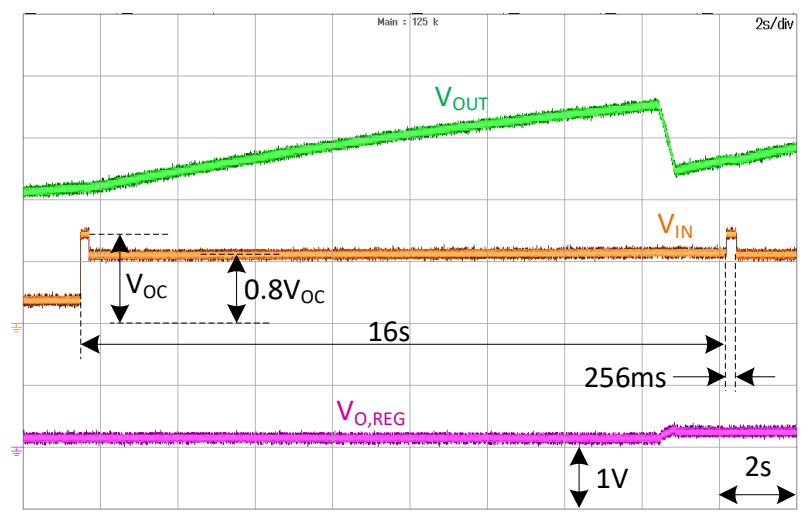

Fig. 6. Zoomed view of the FOCV MPPT algorithm integrated in the BQ25504 PMIC.

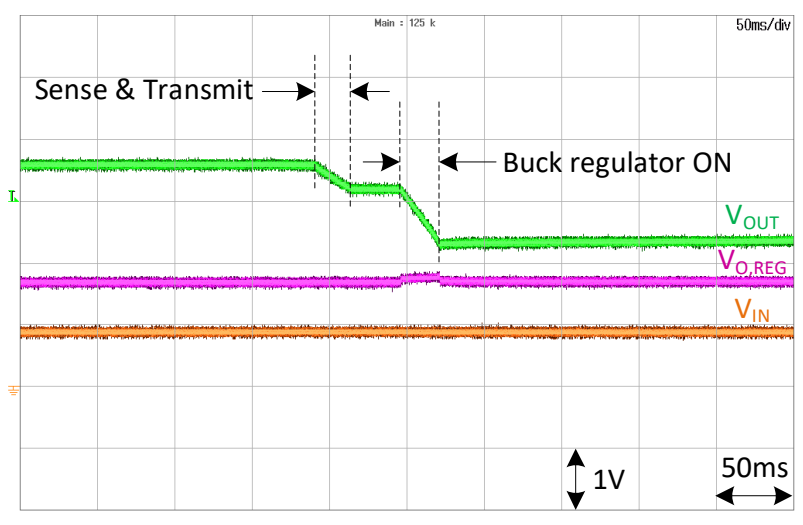

Fig. 7. Important events at each load cycle (zoomed).

average power required $=8.6 \times 10^{4} \mathrm{~nJ} / 16 \mathrm{~s} \approx 5 \mu \mathrm{W}$. Added to that is the continuous power loss of about $3 \mathrm{~V} \times 1 \mu \mathrm{A}$ $=3 \mu \mathrm{W}$ due to the leakage/quiescent currents (assumed as 1 $\mu \mathrm{A})$ in the additional MPPT circuitry $\left(S_{1}, S_{2}\right)$. Therefore, a total of $8 \mu \mathrm{W}$ of power overhead is needed to implement the proposed MPPT method. In comparison, the BQ25504 has a preset $256 \mathrm{~ms}$ FOCV sampling period that is repeated every $16 \mathrm{~s}$ [18], which means it wastes $500 \mu \mathrm{W} \times 0.256 / 16=8$ $\mu \mathrm{W}$ of power for harvesting energy from a cell with $500 \mu \mathrm{W}$ average power.

In this example, the proposed method and the FOCV method

TABLE II

ENERGY REQUIRED TO IMPLEMENT MCU-ASSISTED MPPT

\begin{tabular}{lll}
\hline Task & Time & Energy required \\
\hline Sense $V_{\text {OUT }}$ twice and calculate $V_{\mathrm{MPP}}$ & $95 \mathrm{~ms}$ & $\begin{array}{l}3 \mathrm{~V} \times 0.3 \mathrm{~mA} \times 95 \mathrm{~ms} \\
=8.5 \times 10^{4} \mathrm{~nJ}\end{array}$ \\
Charge $C_{\mathrm{REF}}(=10 \mathrm{nF})$ to $V_{\mathrm{MPP}}(=1 \mathrm{~V}$, say $)$ & $5 \mathrm{~ms}$ & $\begin{array}{l}0.5 \times 10 \mathrm{nF} \times(1 \mathrm{~V})^{2} \\
=5 \mathrm{~nJ}\end{array}$ \\
DAC module active & $5 \mathrm{~ms}$ & $\begin{array}{l}3 \mathrm{~V} \times 0.05 \mathrm{~mA} \times 5 \mathrm{~ms} \\
=750 \mathrm{~nJ}\end{array}$ \\
& &
\end{tabular}

have similar effective power consumption figures. The proposed method will produce a net energy gain if the harvester average power is larger than $500 \mu \mathrm{W}$, and/or if the MPP of the harvester is not well represented by a fixed open-circuit voltage.

\section{CONCLUSIONS}

A COTS component-based dynamic MPPT method for low power energy harvesting WSN applications is demonstrated in this paper. With minimum extra components and less than $10 \mu \mathrm{W}$ power overhead, the proposed method overcomes the shortcomings of FOCV method in the low-milliwatt power realm and does not require additional current and voltage sensors. The analysis and experimental demonstration indicate the speed and accuracy of MPPT can be traded against power consumption by adjusting the step size and the frequency of the MPPT cycle.

\section{REFERENCES}

[1] D. Newell and M. Duffy, "Review of power conversion and energy management for low-power, low-voltage energy harvesting powered wireless sensors," IEEE Trans. Power Electron., vol. 34, no. 10, pp. 9794-9805, Oct 2019.

[2] Z. J. Chew, T. Ruan, and M. Zhu, "Power management circuit for wireless sensor nodes powered by energy harvesting: On the synergy of harvester and load," IEEE Trans. Power Electron., vol. 34, no. 9, pp. 8671-8681, Sep. 2019.

[3] H. Abu-Rub, M. Malinowski, and K. Al-Haddad, Power electronics for renewable energy systems, transportation and industrial applications. John Wiley \& Sons, 2014.

[4] B. Liu, S. Duan, F. Liu, and P. Xu, "Analysis and improvement of maximum power point tracking algorithm based on incremental conductance method for photovoltaic array," in 2007 7th International Conference on Power Electronics and Drive Systems, Nov 2007, pp. 637-641.

[5] "LTC3105: 400mA step-up dc/dc converter with maximum power point control and 250mV start-up," Linear Technology, 2010.

[6] "BQ25504: ultra low-power boost converter with battery management for energy harvester applications," Texas Instruments, June 2015.

[7] "BQ25570: nano power boost charger and buck converter for energy harvester powered applications," Texas Instruments, Dec 2018.

[8] "SPV1050: ultralow power energy harvester and battery charger," STMicroelectronics, May 2018.

[9] W. Swiegers and J. H. R. Enslin, "An integrated maximum power point tracker for photovoltaic panels," in IEEE International Symposium on Industrial Electronics. Proceedings. ISIE'98 (Cat. No.98TH8357), vol. 1, July 1998, pp. 40-44 vol.1.

[10] D. P. Hohm and M. E. Ropp, "Comparative study of maximum power point tracking algorithms using an experimental, programmable, maximum power point tracking test bed," in Conference Record of the Twenty-Eighth IEEE Photovoltaic Specialists Conference - 2000 (Cat. No.00CH37036), Sept 2000, pp. 1699-1702.

[11] N. Femia, G. Petrone, G. Spagnuolo, and M. Vitelli, "Optimization of perturb and observe maximum power point tracking method," IEEE Trans. Power Electron., vol. 20, no. 4, pp. 963-973, July 2005.

[12] S. Liu and R. A. Dougal, "Dynamic multiphysics model for solar array," IEEE Trans. Energy Conv., vol. 17, no. 2, pp. 285-294, June 2002.

[13] “CC430 family user's guide," Texas Instruments, Jan 2013.

[14] K. Ali and D. J. Rogers, "An orientation-independent multi-input energy harvesting wireless sensor node," IEEE Trans. Ind. Electron., vol. 68, no. 2, pp. 1665-1674, 2021.

[15] "MSP430x4xx: family user's guide," Texas Instruments, April 2013.

[16] B. Ivey, R. Hegde, and A. Bhat, "AN1267: extreme low-power (XLP) PIC microcontrollers: An introduction to microchip's low-power devices," Microchip Technology Inc., July 2016.

[17] "Capacitance and dissipation factor measurement of chip multilayer ceramic capacitors," Murata Mfg. Co. Ltd., Oct 2006.

[18] U. Lyles and Y. Ramadass, "BQ25504 optimization of mppt algorithm," Texas Instruments, Mar 2012. 